\title{
Statistical mechanics of aggregation and crystallization for semiflexible polymers
}

\author{
Christoph Junghans ${ }^{1,2}$ (a), Michael Bachmann ${ }^{1,3}$ (b) and Wolfhard Janke ${ }^{1}$ (c) \\ 1 Institut für Theoretische Physik and Centre for Theoretical Sciences (NTZ), Universität Leipzig - Postfach 100 920, \\ D-04009 Leipzig, Germany, EU \\ 2 Max-Planck-Institut für Polymerforschung - Ackermannweg 10, D-55128 Mainz, Germany, EU \\ Institut für Festkörperforschung, Theorie II, Forschungszentrum Jülich - D-52425 Jülich, Germany, EU
}

PACS $05.10 .-\mathrm{a}$ - Computational methods in statistical physics and nonlinear dynamics
PACS $87.15 . \mathrm{A}-$ - Theory, modeling, and computer simulation
PACS $87.15 . \mathrm{Cc}$ - Folding: thermodynamics, statistical mechanics, models, and pathways

\begin{abstract}
By means of multicanonical computer simulations, we investigate thermodynamic properties of the aggregation of interacting semiflexible polymers. We analyze a mesoscopic beadstick model, where nonbonded monomers interact via Lennard-Jones forces. Aggregation turns out to be a process, in which the constituents experience strong structural fluctuations, similar to peptides in coupled folding-binding cluster formation processes. In contrast to a recently studied related proteinlike hydrophobic-polar heteropolymer model, aggregation and crystallization are separate processes for a homopolymer with the same small bending rigidity. Rather stiff semiflexible polymers form a liquid-crystal-like phase, as expected. In analogy to the heteropolymer study, we find that the first-order-like aggregation transition of the complexes is accompanied by strong system-size dependent hierarchical surface effects. In consequence, the polymer aggregation is a phase-separation process with entropy reduction.
\end{abstract}

Cluster formation and crystallization of polymers are processes which are interesting for technological applications, e.g., for the design of new materials with certain mechanical properties or nanoelectronic organic devices and polymeric solar cells. From a biophysical point of view, the understanding of peptide oligomerization, but also the (de)fragmentation in semiflexible biopolymer systems like actin networks is of substantial relevance. This requires a systematic analysis of the basic properties of the polymeric cluster formation processes, in particular, for small polymer complexes on the nanoscale, where surface effects are competing noticeably with structure-formation processes in the interior of the aggregate.

A further motivation for investigating the aggregation transition of semiflexible homopolymer chains derives from the intriguing results of our recent study of a similar aggregation process for peptides [1,2], which were modeled as heteropolymers with a sequence of two types of monomers, hydrophobic $(A)$ and hydrophilic ones $(B)$. By

\footnotetext{
(a) E-mail: junghans@mpip-mainz .mpg ·de

(b) E-mail: m.bachmann@fz-juelich.de

(c) E-mail: Wolfhard.Janke@itp.uni-leipzig.de
}

specializing the previously employed heteropolymer model to the apparently simpler homopolymer case, we aim by comparison at isolating those properties which are mainly driven by the sequence properties of heteropolymers. In fact, while in both cases the aggregation transition is a phase-separation process, we will show below that for homopolymers the aggregation and crystallization (if any) are separate conformational transitions - unlike our study of heteropolymer aggregates where they were found to coincide [1,2]. The physical origin causing these differences will be explained within the microcanonical formalism [3, 4, which proves [1,2] to be particularly suitable for this type of problem.

We thus consider the same model as in 1, 2, but here we assume that all monomers $i_{\mu}=1, \ldots, N^{(\mu)}$ of the $\mu$ th chain $(\mu=1, \ldots, M)$ at positions $\mathbf{x}_{i_{\mu}}$ are hydrophobic $(A)$. The bonds between adjacent monomers are taken to be rigid (bead-stick model) and pairwise interactions among nonbonded monomers are modeled by a LennardJones potential

$$
V_{\mathrm{LJ}}\left(r_{i_{\mu} j_{\nu}}\right)=4\left[r_{i_{\mu} j_{\nu}}^{-12}-r_{i_{\mu} j_{\nu}}^{-6}\right]
$$


where $r_{i_{\mu} j_{\nu}}=\left|\mathbf{x}_{i_{\mu}}-\mathbf{x}_{j_{\nu}}\right|$ is the distance between monomers $i_{\mu}$ and $j_{\nu}$ of the $\mu$ th and $\nu$ th chain, respectively. In the peptide model, the coefficient of the van der Waals contribution $\propto r^{-6}$ would depend on the type of monomers involved [1,2. Intra-chain $(\mu=\nu)$ and inter-chain $(\mu \neq \nu)$ contacts are not distinguished energetically. The semiflexibility of a chain is described by the bending energy

$$
E_{\text {bend }}^{(\mu)}=\kappa \sum_{i_{\mu}}\left(1-\cos \vartheta_{i_{\mu}}\right),
$$

where $0 \leq \vartheta_{i_{\mu}} \leq \pi$ is the bending angle formed by the monomers $i_{\mu}, i_{\mu}+1$, and $i_{\mu}+2$. For the comparison with our recent heteropolymer aggregation studies [1,2], we choose in most simulations a bending rigidity $\kappa=0.25$, which is at the rather floppy end of semiflexibility. Thus, the single-chain energy reads

$$
E^{(\mu)}=E_{\text {bend }}^{(\mu)}+\sum_{j_{\mu}>i_{\mu}+1} V_{\mathrm{LJ}}\left(r_{i_{\mu} j_{\mu}}\right)
$$

and the total energy of the polymer system is given by

$$
E=\sum_{\mu} E^{(\mu)}+\sum_{\mu<\nu} \sum_{i_{\mu}, j_{\nu}} V_{\mathrm{LJ}}\left(r_{i_{\mu} j_{\nu}}\right) .
$$

All chains are assumed to have the same degree of polymerisation, i.e., the same number of monomers, $N^{(\mu)}=N$, $\mu=1, \ldots, M$.

We have performed multicanonical computer simulations [5] for different system sizes. The multicanonical weights were calculated recursively [6] in 50 iterations with $5 \times 10^{6} \times M^{2}$ single updates (spherical pivot rotations [7, semilocal crankshaft moves 2]) each. The production run with fixed multicanonical weights included $5 \times 10^{8} \times M^{2}$ single updates. The generic result obtained from these simulations is a precise estimate for the density of states $g(E)$ or microcanonical entropy $S(E)=k_{B} \ln g(E)$, which will be central in the second part of our data analysis.

From a more standard canonical perspective, conformational transitions between structural macrostates are signalized by peaks in the temperature-dependent fluctuations of energy, i.e., the specific heat per monomer

$$
c_{V}=\left(\left\langle E^{2}\right\rangle-\langle E\rangle^{2}\right) / N_{\text {tot }} k_{B} T
$$

where $N_{\text {tot }}=N M$ and $k_{B}=1$ in our units. Knowing $g(E)$, this can be straightforwardly computed for any temperature $T$. In Fig. 1(a), the specific-heat curve for a system of two identical semiflexible polymers $\left(2 \times A_{13}\right)$ is compared with the energetic fluctuations of a single chain $\left(1 \times A_{13}\right)$. The single chain exhibits a very weak coil-globule collapse transition (shoulder near $T \approx 0.88$ ), whereas the crystallization near $T \approx 0.24$ is a pronounced, separate process. The thermodynamic phase behavior of single semiflexible polymers in solvent has already been subject of numerous studies, with particular focus on stiffness and finite chain length effects 8 10, where it was shown that the globule-solid transition is more influenced by stiffness effects than the coil-globule transition. In particular, for longer chains, it was found that, depending on the stiffness, single collapsed semiflexible polymers form spherical, ellipsoidal, and disklike globules, as well as toroids 10. Our first result for the semiflexible multiple-chain system read off from Fig 1(a) is that aggregation and collapse are not separate processes (near $T \approx 0.97$ ), similar to the corresponding heteropolymer system [1,2].

At about $T \approx 0.24$ (close to the single-chain freezing temperature), the multiple-chain homopolymer complex crystallizes in a separate process. This is in strong contrast to the heteropolymer systems, where aggregation, collapse, and crystallization (hydrophobic-core formation) is a single-step process [1,2]. In Fig. 1(a), we have also included a comparison with a two-chain system with much stronger bending rigidity $\kappa=10$. As expected, there is a single aggregation transition near the temperature, where the system of less stiff semiflexible polymers with $\kappa=0.25$ collapses. However, a further crystallization process at lower temperatures does not occur: There is no globular (liquid) pseudophase of defragmented relatively stiff semiflexible polymers.

Of course, in finite and small polymer systems conformational macrostates form no "phases" in a strict thermodynamic sense. We hence call the stable macrostates in the following "pseudophases" in order to emphasize the difference. Nonetheless, by introducing a "phase" separation parameter

$$
\Gamma^{2}=\frac{1}{2 M^{2}} \sum_{\mu, \nu=1}^{M}\left(\mathbf{r}_{\mathrm{COM}}^{(\mu)}-\mathbf{r}_{\mathrm{COM}}^{(\nu)}\right)^{2},
$$

where $\mathbf{r}_{\mathrm{COM}}^{(\mu)}=\sum_{i_{\mu}=1}^{N} \mathbf{r}_{i_{\mu}} / N$ [2] is the center of mass of the polymers, a clear discrimination of the different pseudophases can be made. Small values of $\Gamma$ correspond to aggregated and higher values to fragmented conformations. In Fig. 1 (b), the canonical expectation value $\langle\Gamma\rangle$ and its fluctuation $d\langle\Gamma\rangle / d T$ are plotted. The peak position of $d\langle\Gamma\rangle / d T$ coincides nicely with the corresponding peak temperature of the specific heat and thus signals the aggregation transition.

In Fig. 2 the aggregation parameter and its fluctuations are shown for different system sizes of up to four chains. The peak shifts towards higher temperatures and gets sharper with increasing number of chains, since intra-chain and inter-chain monomer-monomer contacts are not energetically distinguished. The hypothetic maximal total number of intrinsic contacts is $n_{\text {intra }}^{\max }=$ $M(N-2)(N-1) / 2 \sim M N^{2}=N N_{\text {tot }}$, and for the maximally possible number of inter-chain contacts one has $n_{\text {inter }}^{\max }=M(M-1) N^{2} / 2 \sim M^{2} N^{2}=N_{\text {tot }}^{2}$ [1]. For large $M$, the relative fraction $r_{\text {inter }}$ of the inter-chain contacts,

$$
\begin{aligned}
r_{\text {inter }} & =\frac{n_{\text {inter }}^{\max }}{n_{\text {intra }}^{\max }+n_{\text {inter }}^{\max }} \\
& =1-\frac{1}{M}\left(1-\frac{1}{N}\right)\left(1-\frac{2}{N}\right)+\mathcal{O}\left(\frac{1}{M^{2} N}\right)
\end{aligned}
$$




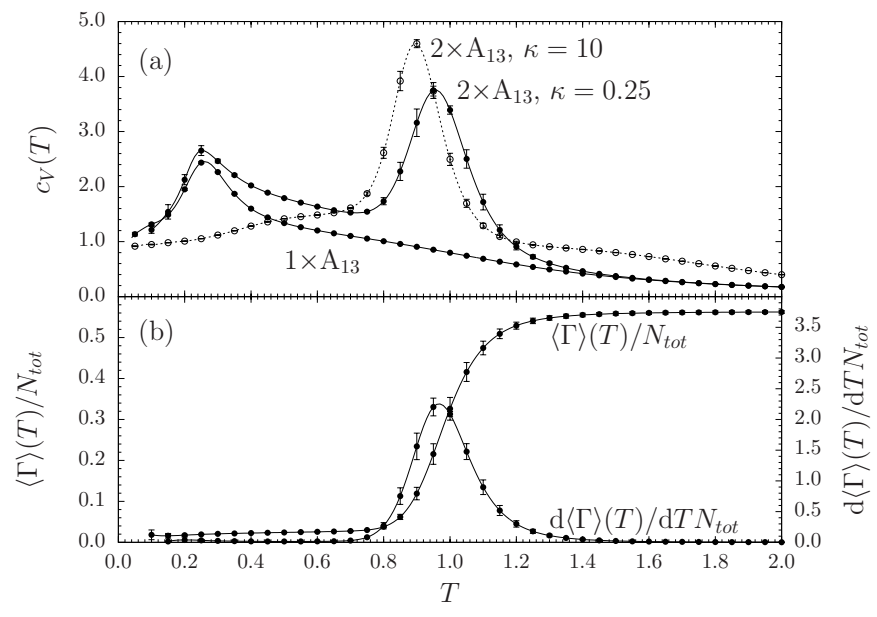

Fig. 1: (a) Specific heat per monomer as a function of temperature for a single semiflexible homopolymer with 13 monomers $\left(1 \times A_{13}, \kappa=0.25\right)$ and for systems of two such chains $\left(2 \times A_{13}\right)$ with different bending rigidities. (b) Canonical expectation value $\langle\Gamma\rangle$ and fluctuation $d\langle\Gamma\rangle / d T$ of the aggregation parameter $\Gamma$, defined in Eq. (6), for the two-chain system with $\kappa=0.25$.

behaves like $r_{\text {inter }} \sim 1-M^{-1}$, i.e., the relative influence of inter-chain contacts increases rapidly towards unity with the number of chains. In consequence, aggregation dominates over collapse of the individual chains. Even for the two-chain system $2 \times A_{13}$ this estimate is reasonable: The energy of the lowest-energy conformation we found numerically is $E^{(\min )} \approx-83.61$ and the contribution of the inter-chain contacts is $E_{\text {inter }}^{(\min )} \approx-50.20 \approx r_{\text {inter }}^{(\min )} E^{(\min )}$ with $r_{\text {inter }}^{(\min )} \approx 0.60$. This coincides nicely with the corresponding value of the above contact ratio, $r_{\text {inter }} \approx 0.56$. In fact, considering energetic and structural fluctuations of the larger systems with three and four chains, we have not found indications for an additional collapse transition at temperatures higher than the aggregation transition.

Below the aggregation temperature, the entropic loss of the individual chains is overcompensated by the energetic gain of forming a joint globular aggregate. However, the entropic change while passing the aggregation transition is noticeably smaller than what we found recently for heteropolymer systems, where no separate freezing transition occurs 2. In the intermediate fluid "globular" pseudophase, the aggregate of the homopolymers thus behaves like a single chain of length $M N$. Consequently, reducing the temperature, the aggregate optimizes the monomer arrangements in order to maximize energetic contacts. Indicated by the peak in the specific heat near $T \approx 0.2$ (see Fig. 1 and also Ref. [7), the small globular aggregates freeze into spherical amorphous structures with a maximum number of inter-chain contacts. For rather stiff semiflexible polymers (as our example with $\kappa=10$ ), however, a separate freezing transition does not occur and the peak of $c_{V}$ near $T \approx 0.9$ indicates a single-step transition from rod-like coils to a liquid-crystal-like phase [10].

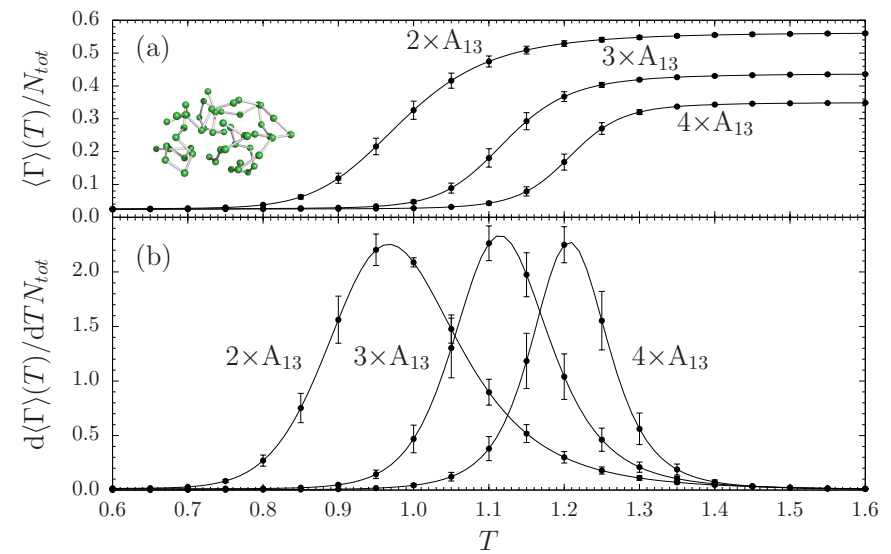

Fig. 2: (a) Canonical expectation values and (b) fluctuations of the aggregation parameter $\Gamma$ for multi-chain systems with $\kappa=0.25$. In (a), also an exemplified globular $4 \times A_{13}$ aggregate is depicted.

In our recent studies of heteropolymer aggregation [1], we also observed a pronounced single transition of a different nature. In this case, the formation of a heteropolymer complex consisting of different chains with compact hydrophobic core is roughly a single-step process, because hydrophobic-core formation ("freezing") favors conformations with very small entropy. For semiflexible homopolymer systems, we find in the rather floppy limit that the freezing temperature $(T \approx 0.2$, for single chains or globular aggregates with $\kappa=0.25)$ is almost identical with the aggregation temperature for heteropolymer systems [1,2]. This coincidence in the behavior of these different systems is due to the formation of a single, very compact hydrophobic domain (the monomers of the interacting homopolymers are as hydophobic as the A-type monomers of the heteropolymer) which maximizes the number of energetic contacts. Stiff homopolymers, on the other hand, cannot form a maximally compact hydrophobic core and hence do not crystallize in a separate transition.

For a deeper understanding of the aggregation transition, we now analyze entropic effects accompanying this transition in the microcanonical ensemble for an isolated system of multiple semiflexible chains with $\kappa=0.25$. The microcanonical entropies per monomer, $s=S / N_{\text {tot }}$, are shown in Fig. 3(a) as functions of the total energy per monomer, $e=E / N_{\text {tot }}$. The reciprocal caloric temperature, $T^{-1}(E)=\partial S / \partial E$, is plotted in Fig. 3(b). The plots reveal an exciting phenomenon: Increasing the energy entails a reduction of temperature in the transition region, known as the backbending effect [3]. This signals a phaseseparation process 1,2, which is caused by surface effects reducing the entropy which, in an isolated system, results in a decrease of temperature by increasing the total system energy [3,4,12] 15. This phenomenon is called "backbending effect", because the caloric temperature curve changes in the transition region its monotonic behavior with increasing total energy [3]. For truly isolated systems, this 


\begin{tabular}{ccccccc}
\hline \hline System & $T_{\text {agg }}$ & $\Delta s_{\text {surf }}$ & $e_{\text {agg }}$ & $e_{\text {frag }}$ & $\Delta q$ & $\Delta q / T_{\text {agg }}$ \\
\hline $2 \times \mathrm{A}_{13}$ & 0.973 & 0.024 & -1.566 & -0.944 & 0.622 & 0.639 \\
$3 \times \mathrm{A}_{13}$ & 1.118 & 0.016 & -1.730 & -0.831 & 0.899 & 0.804 \\
$4 \times \mathrm{A}_{13}$ & 1.172 & 0.009 & -1.892 & -0.799 & 1.093 & 0.932 \\
\hline \hline
\end{tabular}

Table 1: Aggregation temperatures $T_{\text {agg }}$, relative surface entropies per monomer $\Delta s_{\text {surf }}$, relative aggregation and fragmentation energies per monomer, $e_{\text {agg }}$ and $e_{\text {frag }}$, respectively, latent heat per monomer $\Delta q$, and phase-separation entropy per monomer $\Delta q / T_{\text {agg }}$.

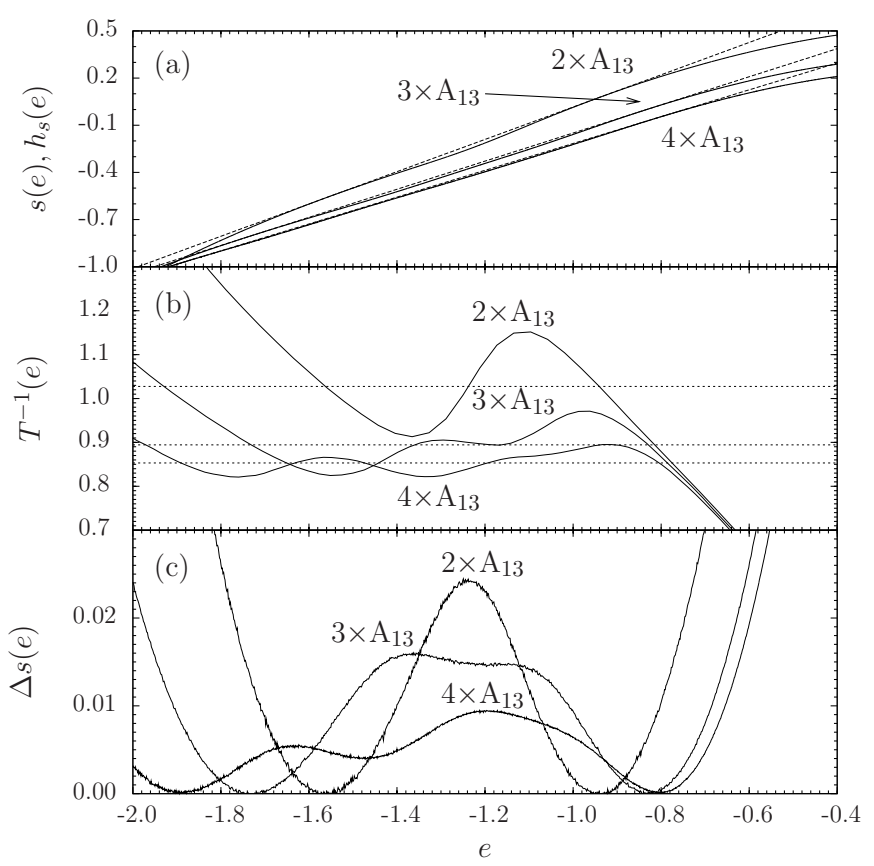

Fig. 3: (a) Microcanonical entropy per monomer $s(e)$ and the Gibbs constructions $h_{s}(E)$ (dashed lines), (b) reciprocal caloric temperatures and Maxwell constructions, and (c) relative surface entropies per monomer $\Delta s_{\text {surf }}$.

is a physical effect which has been verified, e.g., in atomic clustering experiments [16].

In our previous studies of heteropolymer aggregates [1, 2], we took technical advantage of this microcanonical view to identify the peptide aggregation as so-called "coupled binding-folding transition", i.e., the individual heteropolymer chains refold during the binding process and the finally formed aggregate possesses a single compact hydrophobic domain.

By closer inspection of Fig. 3(b), we find for the homoploymer system a hiercharchical substructure caused by these surface effects. The number of oscillations of the curves, increasing with system size, reveals that the aggregation transition is actually a composition of different subprocesses, each of which is an individual phase-separation process. The amplitude of these oscillations decreases with system size showing that these subprocesses comprise a smaller surface-entropic barrier [see Fig. 3(c)].

The $2 \times A_{13}$ system exhibits a single backbending ef- fect as only two chains aggregate. For the three-chain system $3 \times A_{13}$, a different scenario is apparent. In the higher-energy regime, first two chains stick together, and the formation of the three-chain globule is a separate process at lower energies. This hierarchical procedure continues for larger systems as, e.g., for $4 \times A_{13}$. However, the impact of the individual backbending effects is getting weaker and, in the thermodynamic limit, these effects are expected to disappear asymptotically, whereas the firstorder character of this transition remains. The horizontal lines in Fig. 3(b) are the respective Maxwell constructions and define the aggregation temperatures $T_{\text {agg }}$. In the following, we denote the leftmost (rightmost) energy where $T^{-1}(e)=T_{\text {agg }}^{-1}$ as $e_{\text {agg }}\left(e_{\text {frag }}\right)$. In the entropy curves, the Maxwell constructions correspond to concave hulls $h_{s}(e)$ with $\partial h_{s}(e) / \partial e=T_{\text {agg }}^{-1}$ (Gibbs constructions) in the transition regime [see Fig. 3(a)], where entropy is reduced due to surface effects (the convex region of the microcanonical entropy curve is sometimes called "convex intruder" [3]).

For a quantitative analysis, we define $\Delta s(e)=h_{s}(e)-$ $s(e)$ which is plotted in Fig. 3)(c). Within the transition region (i.e., for $e_{\text {agg }} \leq e \leq e_{\text {frag }}$ ), the peak height $\Delta s_{\text {surf }}=\max _{e_{\text {agg }} \leq e \leq e_{\text {frag }}} \Delta s(e)$ defines the surface entropy. The energetic width of the phase-coexistence regime is the latent heat per monomer, $\Delta q=e_{\text {frag }}-e_{\text {agg }}=$ $\left.T_{\text {agg }}\left[s\left(e_{\text {frag }}\right)-s\left(e_{\text {agg }}\right)\right]\right)$. Thus the entropic phase separation barrier $\Delta q / T_{\text {agg }}$ should survive in the thermodynamic limit, if the aggregation of semiflexible polymers is a first-order-like phase-separation process with coexistence of aggregates and fragments.

Values for these quantities are listed in Table 1 for the three polymer systems considered in our study. We find that with increasing system size the surface entropies $\Delta s_{\text {surf }}$ decrease and thus the influence of surface effects is getting weaker. On the other hand, the latent heat per monomer increases, supporting the first-order character of the aggregation transition.

We have shown in this Letter that the aggregation of interacting semiflexible polymers is a first-order phaseseparation process. For two reasons, we focused on small systems with up to four chains with 13 monomers in a cubic box of edge length $L=60$. The first reason is that one of the primary goals of this study was the unraveling of underlying mechanisms in multiple-chain aggregation processes. This also includes the identification of sequentially ordered, i.e., hierarchical, subphase transitions ac- 
companying the phase separation process of aggregation. As our microcanonical analysis revealed, these transitions exhibit peculiarities compared to thermodynamic phase transitions, which are due to dominant entropic surface effects in the small systems considered here. The surface changes at the interface of co-existing aggregates and fragments let the microcanonical temperature decrease while energy increases, whereas far away from the transition region the more intuitive behavior is observed: temperature increases with energy. Because of the monotonic change of the microcanonical temperature in the transition region, this phenomenon is called "backbending". A second reason for restricting ourselves to small systems is the demand for a particularly high accuracy needed for the precise estimation of the density of states which is the basis for the microcanonical analysis. The observed surface effects are expected to vanish in the thermodynamic limit 2. However, for molecular small-scale applications based on the interplay of only a few molecules and the understanding of biomolecular aggregation processes, the thermodynamics of finite-size effects is relevant.

Our precise microcanonical analysis revealed that for homopolymers the aggregation transition is accompanied by hierarchical backbending effects. We found that after aggregation, complexes of rather floppy semiflexible polymers behave like globules in the liquid regime and freeze at low temperatures in a separate process. In contrast, for rather stiff homopolymers, no separate freezing transition is observed, in coincidence with former results [10]. The overall transition behavior of semiflexible homopolymers is also different compared to the previously studied heteropolymer model 1, 2, which only differs in the sequential disorder of hydrophobic and polar monomers, whereas in the homopolymer case all monomers are treated as hydrophobic. However, we found similarities in the transition towards the formation of a single hydrophobic domain, provided the stiffness of the homopolymer chain is sufficiently weak.

The understanding of aggregation and crystallization processes of polymers is a necessary prerequisite for the design of technological applications in material science as, e.g., ordered nanoscopic structures like fibers, nanopores, and channels or amorphous polymeric cells with particular electronic properties. Aggregation processes are also essential in biological systems, where enzymatic and motoric action is mediated by molecular binding processes. Furthermore, molecular cluster formation can also cause disastrous diseases like Alzheimer's, which is yet another reason, why generic features of polymer-polymer interactions are worth being studied.

This work is partially supported by the DFG (German Science Foundation) under Grant Nos. JA 483/24-1/2/3, the Leipzig Graduate School of Excellence "BuildMoNa", and by the Max Planck Society. Support by a NIC supercomputer time grant (No. hlz11) of the Forschungszentrum Jülich is acknowledged.

\section{REFERENCES}

[1] C. Junghans, M. Bachmann, and W. Janke, Phys. Rev. Lett. 97, 218103 (2006).

[2] C. Junghans, M. Bachmann, and W. Janke, J. Chem. Phys. 128, 085103 (2008).

[3] D. H. E. Gross, Microcanonical Thermodynamics (World Scientific, Singapore, 2001).

[4] W. Thirring, Z. Physik 235, 339 (1970).

[5] B. A. Berg and T. Neuhaus, Phys. Lett. B 267, 249 (1991); Phys. Rev. Lett. 68, 9 (1992).

[6] W. Janke, Physica A 254, 164 (1998); B. A. Berg, Fields Inst. Commun. 26, 1 (2000).

[7] M. Bachmann, H. Arkın, and W. Janke, Phys. Rev. E 71, 031906 (2005).

[8] S. Doniach, T. Garel, and H. Orland, J. Chem. Phys. 105, 1601 (1996); J. P. K. Doye, R. P. Sear, and D. Frenkel, J. Chem. Phys. 108, 2134 (1998).

[9] V. A. Ivanov, W. Paul, and K. Binder, J. Chem. Phys. 109, 5659 (1998).

[10] M. R. Stukan, V. A. Ivanov, A. Yu. Grosberg, W. Paul, and K. Binder, J. Chem. Phys. 118, 3392 (2003).

[11] Due to excluded volume and optimal space-filling constraints, these numbers need to be scaled by about $q / N_{\text {tot }}$, where $q$ is the effective coordination number (which is, e.g., $q \approx 10.0$ for a perfect fcc crystal).

[12] D. J. Wales and R. S. Berry, Phys. Rev. Lett. 73, 2875 (1994).

[13] W. Janke, Nucl. Phys. B (Proc. Suppl.) 63A-C, 631 (1998).

[14] S. Hilbert and J. Dunkel, Phys. Rev. E 74, 011120 (2006).

[15] H. Behringer and M. Pleimling, Phys. Rev. E 74, 011108 (2006).

[16] M. Schmidt, R. Kusche, T. Hippler, J. Donges, W. Kronmüller, B. von Issendorff, and H. Haberland, Phys. Rev. Lett. 86, 1191 (2001). 